\title{
Strates
}

STRATES Matériaux pour la recherche en sciences sociales

6 | 1992

La question de l'environnement : naissance d'un débat en Pologne

\section{La diaspora égéenne : récit d'une femme}

\section{Frosso Vassilikioti}

\section{(2) OpenEdition}

10 Journals

\section{Édition électronique}

URL : https://journals.openedition.org/strates/3953

DOI : $10.4000 /$ strates.3953

ISSN : 1777-5442

Éditeur

Laboratoire Ladyss

\section{Édition imprimée}

Date de publication : 31 mars 1992

ISSN : 0768-8067

Référence électronique

Frosso Vassilikioti, «La diaspora égéenne : récit d'une femme », Strates [En ligne], 6 | 1992, mis en ligne le 22 novembre 2007, consulté le 21 septembre 2021. URL : http://journals.openedition.org/strates/ 3953 ; DOI : https://doi.org/10.4000/strates.3953

Ce document a été généré automatiquement le 21 septembre 2021.

Tous droits réservés 


\title{
La diaspora égéenne : récit d'une femme
}

\author{
Frosso Vassilikioti
}

L'île sans terre

1 Mon grand-père maternel disait toujours que Dieu avait voulu faire du bien aux îles de l'Égée, et qu'il avait tamisé leur terre. Et après qu'il eut bien tamisé, tout ce qui restait, toutes les pierres, il les avait versées sur Mykonos! Oui, Mykonos a toujours été très pauvre, parce qu'il n'y avait pas de terre, pas d'eau.

2 Et pourtant les gens qui savent ont toujours dit qu'ici tous les produits ont une autre saveur, plus intense, parce qu'il n'y a pas d'eau. Mais la vie était très dure pour les paysans. Ils attendaient la pluie... Ils se réveillaient à trois heures du matin - les enfants aussi, garçons et filles -, et ils travaillaient à longueur de journée. Et ils mangeaient mal, il n'y a encore pas si longtemps.

3 Il y avait pu de distinction entre eux, l'un avait un lopin un peu plus grand, l'autre un peu plus petit. Pas de grands propriétaires terriens, des rentiers. Quelques Mykoniates possédaient des terres, la plupart du temps les paysans les payaient en nature, tant de boisseaux de ceci et de cela, l'argent était rare.

4 L'usure, en revanche, était très rentable, et c'est comme cela que quelques-uns ont commencé à faire fortune. Les paysans leur devaient toujours de l'argent, ils leur portaient l'icône, et le bijou de la femme, le peu qu'ils avaient...

5 Il y avait les paysans, et puis les gens du port, des pêcheurs surtout, et des bateliers. Parmi eux, trois ou quatre petits armateurs, des kapétani, des capitaines, qui avaient un caïque, un gros bateau, et qui faisaient du commerce. Ils partaient pour un, deux ou trois ans. Et ils prenaient avec eux des montagnes de pains - c'est pour cela qu'à Mykonos on endort les enfants en leur chantant:

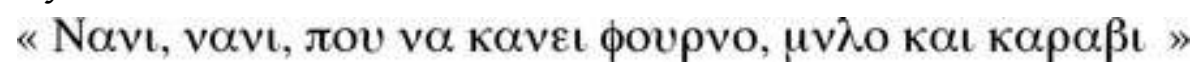

6 (Dodo, dodo, qu'il ait four, moulin et bateau). Ils allaient dans toute la Méditerranée, en mer Noire aussi, et en Roumanie, sur le fleuve, et ils chargeaient... Mais leur activité était centrée ici, ils se mariaient ici, leurs enfants vivaient ici. Et la kapétanissa (la 
femme du capitaine) était responsable des familles des marins, c'est-à-dire qu'elle assurait tout leur entretien. Lorsque les bateaux revenaient, on faisait les comptes, chaque famille devait lui rendre ce qu'elle avait dépensé. Et on faisait des fêtes. La marine de Mykonos était très florissante et il $\mathrm{y}$ a même eu des moments où elle comptait parmi les premières marines de Grèce après Hydra. Et certains de ces « armateurs » étaient très éclairés : Kounéni, par exemple, au début du siècle, avait fait venir une maîtresse d'école qui avait enseigné le français à ses filles, et il avait apporté de France, pour elles, une bibliothèque extraordinaire.

7 Mais les quelques familles importantes, comme les Axiotis, la famille de mon père, ou les Gripari, celle de ma mère, n'avaient pas leur fortune ici. Elles étaient indépendantes de Mykonos, elles n'y vivaient plus, elles venaient comme visiteurs, l'été, parce qu'elles possédaient des maisons. La Grèce n'existait pas en tant que champ d'activité, bien entendu. Que vouliez-vous qu'ils fassent ici ? Ils allaient ailleurs, tous s'en allaient, tous les garçons s'en allaient...

De Constantinople aux « folies russes »

8 Du côté de mon père, c'est une famille où les professions étaient surtout artistiques, ou politiques. Alexandros Axiotis, mon arrière grand-père, était venu de Constantinople au moment de la révolution de 1821. C'est à lui que les Turcs avaient rendu l'Acropole lorsqu'ils étaient partis, parce qu'il était alors gouverneur d'Athènes. Puis il a été nommé gouverneur des Cyclades, il a épousé une Mykoniate et reçu cette maison en dot. Lui-même était originaire des Cyclades : Axiotis veut dire « de Naxos ».

9 Mon arrière grand-père n'a laissé derrière lui... que sa carrière politique. Il a eu un fils, Panagos, mon grand-père, qui est né en 1840. En ce temps là, les Grecs s'expatriaient quand ils pouvaient le faire, l'un partait et, dès qu'il faisait fortune, les autres suivaient. C'était chose facile en Russie, parce qu'on faisait travailler les Russes comme des bêtes. Comme beaucoup de Grecs - et beaucoup de Mykoniates -, Panagos est donc parti en Russie du Sud, où il était marchand. Il y avait alors un très grand commerce de farine avec la mer Noire, et la Russie approvisionnait l'Europe entière. Quand j'entends dire à la télévision que la Russie manque aujourd'hui de farine... Mon grand-père était donc très riche. Il a voulu vivre, il est revenu en Grèce, il a investi son argent. Et il est devenu écrivain. Il y avait cette tradition dans la famille, des bibliothèques de toutes les couleurs, dans toutes les langues, tout ce que vous pouvez imaginer comme littératures, ça les intéressait toujours beaucoup.

10 Mon père, lui, est devenu musicien, compositeur de musique classique. Il a fait ses études à Naples, c'était alors la mode, pour la musique. C'était le premier musicien de la famille. Enfin on ne sait pas... peut-être que du côté de sa mère, qui était Russe! Il ne faisait rien d'autre que de la musique, il avait fondé le conservatoire du Pirée, qu'il dirigeait, il composait, il donnait des concerts. Et il cultivait la terre, il adorait ça : il avait fait cette maison et ce jardin d'Ano Méra ${ }^{1}$ qui était une chose aberrante... Il avait choisi l'emplacement, acheté le terrain, il n'y avait pas de terre là-bas, c'était du rocher. Et comme il avait l'ambition de faire un jardin - qu'il a fait, d'ailleurs, magnifique -, il a fait venir de la terre de Naxos ! Oui, des folies de Russe, il envoyait des caïques à Naxos qui apportaient de la terre à Kalo Livadi, et de là, à dos d'âne, ou à dos de mulet, dans des sentiers inimaginables, parce qu'il n'y avait pas de route, ils ont apporté cette terre à Koukoulou. Et l'été, quand j'étais enfant, nous montions à Ano Méra pour cinq mois, il y avait une vraie caravane, six ou sept ânes, et le piano qui montait sur un cheval plus 
costaud que les autres... Mon père était le plus heureux des hommes. Lui, sa musique, et son jardin...

11 Tout ce qu'il avait fait planter, c'était venu d'ailleurs, il avait fait venir des fleurs de Hollande, d'Espagne des orangers, des citronniers... C'était son rêve... et il a ignoré tout le reste. Et entre temps, la fortune fondait. Pas seulement à cause de ces folies. Venu de Russie avec une grosse fortune, mon grand-père avait fait de mauvais placements. Et lorsque mon père dépensait follement, son père à lui avait cessé d'être riche. Mon père a eu beaucoup de chance... parce qu'il est mort à temps... pour lui, heureux, avant qu'il ne se rende compte qu'il n'y avait plus d'argent...

«L'empire » de Baranovka

Du côté de ma mère, ils étaient d'origine vénitienne ${ }^{2}$. Et ils ont toujours fait du commerce bien sûr, ils n'ont jamais eu de terres. Mon arrière-grand-père maternel, Pierre Gripari, et Marietta Gripari ont eu sept garçons, qui se sont éparpillés là où il y avait de l'argent.

Certains sont allés en Russie au milieu du siècle dernier - exactement comme les Axiotis. Et mon grand-oncle Nicolas, le frère de mon grand-père, est devenu un « richard ». Vers 1880, lui et son frère Démosthène voyageaient en Russie, et dans un train, assis, ils ont entendu deux dames russes raconter que telle famille, qui habitait Paris, avait une étendue immense, immense, qui s'appelait Baranovka. Ces gens s'étaient rendu compte que le gérant les volait, et ils voulaient vendre tout ça. Et je ne sais pas comment oncle Nicolas a fait pour acheter cette propriété, c'était une chose inouïe, une province entière, il y avait là tout ce qu'on peut imaginer, des villages, des terres, de l'agriculture, une fameuse fabrique de porcelaine... ${ }^{3}$ Je me rappelle très bien, parce que c'était spectaculaire... En 1919, il y a eu un baril très grand, qui est arrivé ici, à Mykonos. Il avait été envoyé de Russie, vers la révolution, et il est arrivé là, sur la petite place, il fallait mettre une échelle, il y avait de la paille, et c'était plein de porcelaine, qu'on envoyait à ma mère...

14 À cause de cette histoire de Baranovka, Nicolas Gripari a attiré tous les autres. Il avait lui-même quatre ou cinq fils, et ses frères de nombreux garçons. Et les Grecs, dès qu'ils montaient un peu financièrement, envoyaient tout de suite leurs enfants étudier à l'étranger pour qu'ils deviennent médecins, ingénieurs ou architectes. En France, en Angleterre... pas encore en Amérique - d'ailleurs, c'est sans aucun intérêt culturel. Certains Grecs envoyaient aussi leurs garçons à Marseille, pour apprendre le commerce. Ils voulaient toujours apprendre des autres... Mais un commerçant qui faisait de son fils un commerçant, c'était un petit peu déconsidéré. Les professions libérales, c'était une promotion, bien entendu. Donc Nicolas Gripari a fait venir des frères et des neveux en Russie, et c'est lui qui a fait étudier ces douze ou treize garçons dans les meilleures écoles en Europe. Quant aux filles, on les faisait venir pour un temps, puis elles repartaient en Grèce, elles étaient "instruites "-elles savaient le français, le grec, elles chantaient très bien - et elles se mariaient ici.

Ces familles riches considéraient qu'elles avaient des devoirs très grands envers la famille, les neveux, les cousins, et aussi envers les gens de Mykonos. Il y avait beaucoup de solidarité. Mykonos, c'était très petit, ça ne veut peut-être rien dire, mais tout le monde se connaissait, et les riches, qui avaient besoin de beaucoup de domestiques ou d'employés pour leurs maisons de commerce, emmenaient les gens qui avaient besoin d'un travail, et puis ils les mariaient. Il y avait ainsi toujours une attache avec Mykonos. 

neveux avaient fait leurs vies, leurs études, et oncle Nicolas a fini sa vie ici, à Mykonos, avec sa femme. Mais pour d'autres, une fois la Russie finie, il n'y avait plus d'argent. Alors il ne restait que les mariages. Et je me souviens d'une cousine qu'on avait forcé à épouser un type qui avait fait fortune en Asie Mineure. Ça a été terrible... Elle n'en voulait pas. Ma mère m'a raconté qu'il avait fallu lui faire des piqûres pour la contraindre à l'épouser. C'était en 1919... En 22 il y a eu la catastrophe d'Asie Mineure, et il n'a plus eu un sou.

D'autres épousaient des garçons - ou des filles - dont les familles étaient dans le commerce international. C'est comme cela qu'il y a eu des Gripari en Égypte tout à fait au début du siècle. Pierre, le fils aîné de Nicolas Gripari, était architecte, installé à Alexandrie, très riche aussi, parce qu'il avait épousé une fille de la famille Ralli, Rallibrothers ${ }^{4}$, c'était aussi un empire, c'était presque une dynastie aux Indes. Ils avaient une succursale à Manchester, et mon oncle Pierre, qui était ingénieur civil là-bas, y avait connu Jeanne Ralli. Et beaucoup de garçons sont allés travailler chez les Ralli, un frère de ma mère, par exemple, est allé aux Indes.

\section{Égypt} deux. Alors il l'a enlevée, tout simplement... Et il l'a emmenée en Grèce, à Tinos, dans un village où elle avait des parents - Interpol n'existait pas à cette époque. Elle était enceinte. Il a attendu cinq ans. Et lorsqu'elle est devenue majeure, il l'a épousée, ils sont venus à Mykonos.

19 Ma mère, Maroulina, était leur troisième fille. Sa sœur Marietta était un monstre de méchanceté, sa sœur Anetta un ange. Marietta la mauvaise est allée vivre en Égypte, Anetta l'ange est allée en Égypte.

Alexandrie : « richards » et bourgeois

20 À la mort de mon père, ma mère et moi nous sommes donc parties vivre en Égypte, et les sœurs de ma mère se sont occupées de nous. C'était en 1924, j'avais douze ans. Elles m'ont mise en pension dans une très bonne école. Et ma mère a habité chez Anetta. Son mari était représentant de commerce. Sa sœur Marietta, c'était un tout autre niveau. Son mari était l'avocat de tous les «richards » d'Alexandrie. Elle avait une maison de vingt-six pièces, pour... cinq personnes, dont trois enfants. Elle sortait rarement, mais elle achetait, elle achetait... des robes, des fourrures, des toilettes, des chapeaux, des souliers, c'était la manie d'amasser. En été, tous partaient en Europe, en Suisse, Marietta, ses trois gosses, son chauffeur et sa femme de chambre. Elle louait une suite dans un grand hôtel, pour l'été. Et pourtant elle n'était pas parmi les plus riches de la ville.

21 À cette époque, en Égypte, le coton c'était comme le pétrole aujourd'hui, un trafic extraordinaire. Tout le gros commerce du coton était entre les mains des Grecs. Après le départ des Anglais, les Égyptions n'avaient pas été à même de prendre le pouvoir et l'économie en mains, parce qu'ils avaient été tenus dans un tel niveau... Alors les Grecs avaient continué. Leur domaine, c'était la bourse. Ils investissaient de l'argent dans ce qu'on appelait les fidania, ces étendues immenses de coton, où travaillaient des ouvriers égyptiens totalement exploités, méprisés, qui vivaient dans des sortes de huttes en bouse. Ils spéculaient sur ces fidania, il y avait des faillites spectaculaires, 
deux suicides pendant que j'étais là. Et la plupart des fortunes n'arrivaient pas à tenir sur plusieurs générations.

Tout à fait à la tête, il y avait cinq ou six familles. Si vous aviez vu les maisons qu'ils avaient... des palais, un train de vie extraordinaire... Les très grosses fortunes, c'était les Bénakis ${ }^{5}$ et les Salvagos ${ }^{6}$. Ils avaient un carré entier au cœur de la ville, des maisons magnifiques, dans des parcs à eux. C'était une caste, les " richards ». Ils vivaient entre eux, ils étaient comme une famille, ils se mariaient entre eux. Ils étaient tellement unis entre eux qu'ils tombaient forcément amoureux... là où il fallait.

Quand ces familles avaient des fils, il fallait qu'ils continuent les affaires, toujours. On ne les envoyait pas étudier quelque chose, il n'y a jamais eu un avocat, ou un ingénieur, ou un architecte parmi eux, non. Le plus prestigieux, pour ces gens-là, c'était la vie mondaine, il y avait beaucoup d'argent qui allait dans des dîners, des bals, des choses comme ça. Ils étaient fiers d'être Grecs, parce qu'ils tenaient le haut du pavé. Mais ils voulaient faire voir qu'ils étaient aussi Européens. Alors ils dépensaient en toilettes, en meubles, en objets pour la maison. Il y avait des représentations des grandes maisons de mode de Paris. Les meubles, c'était des meubles français, tous les grands magasins parisiens avaient des succursales, les Galeries Lafayette, le Louvre, le Bon Marché, et tous les employés de commerce parlaient français. Ils dépensaient beaucoup en voyages, ils allaient en France, à Paris, sur la Côte d'Azur, en Suisse. C'était de l'argent vite gagné, vite dépensé... et vite perdu.

Et ils faisaient de la bienfaisance. Des écoles - pas pour les riches, les riches n'allaient pas à l'école communale. Des orphelinats, des hospices pour les vieillards, une église, ce genre de choses. C'était d'abord pour laisser leur nom : Benaki likio (lycée Bénakis), Salvago scholi (école Salvagos). Et Bénakis a laissé aussi un musée à Athènes. Il y avait une émulation entre eux, l'un faisait une école, l'autre un orphelinat, et ainsi de suite, pour le nom. C'était des vies entières à la conquête d'un prestige social... Et ce n'était pas des gens qui avaient été privés. Je pense qu'ils avaient un complexe, ces gens-là, parce qu'ils n'étaient pas des ducs ou des marquis. Alors ils tâchaient de se faire valoir.

Il y avait des "richards", les grandes familles, avec les grosses fortunes, les magnifiques maisons, les parcs, beaucoup de prestige. Et puis après cela il y avait des gens qui exerçaient une profession, ou qui faisaient du commerce, mais pas sur la même échelle. C'était des bourgeois - rien à voir avec ces «richards »- dont les fils devenaient architectes, ou ingénieurs, ou dentistes, ou médecins. Des bourgeois, une bonne classe, des gens bien. Les « richards ", eux, n'avaient pas de métier. C'était des gens qui maniaient l'argent, simplement. Les uns et les autres se fréquentaient très bien, ils se mélangeaient mondainement, mais pas au point de se marier entre eux. C'était coupé.

Avec leurs parents de Grèce, les Grecs d'Égypte se sentaient un petit peu protecteurs, un peu paternalistes. C'était plus prestigieux d'être un Grec d'Alexandrie... La Grèce ne pouvait pas offrir des occasions pareilles. D'ailleurs ces gens-là n'avaient pas d'intérêts en Grèce. Et c'était la mode alors, les garçons désargentés d'Athènes cherchaient à épouser des filles d'Alexandrie - après Nasser, ça a été exactement le contraire, ce sont les garçons désargentés d'Égypte qui ont couru après les filles riches d'Athènes... Ils n'avaient plus d'argent, mais ils revenaient avec un nom...

À Alexandrie, il y avait aussi quelques familles juives ou coptes très fortunées qui faisaient le commerce de coton. Avec les Juifs, les Grecs entretenaient des rapports d'amitié. Ils ne faisaient aucune distinction entre eux, ils se rencontraient à la bourse, 
ils se recevaient mondainement, ils étaient très amis. Les Grecs n'étaient pas antisémites.

Mais il y avait très peu de mariages mixtes. Le mariage d'une Grecque avec un Syrien, c'était tout juste accepté, si le garçon appartenait déjà à une classe sociale, et s'il était orthodoxe. Mais musulman jamais, c'était impensable. Pas seulement à cause de la religion, mais du mépris des Égyptions, pour le peuple, qui continuait. Ils se sentaient supérieurs, comme dans les colonies. Personne ne parlait l'arabe, ou très peu, pour s'adresser aux domestiques. Et même, les domestiques de bonnes maisons apprenaient le français.

Alexandrie était tout à fait une ville européenne, rien à faire avec le pays. C'était une petite ville, et pourtant qu'est-ce qu'elle contenait! Au point de vue intellectuel, c'était absolument une succursale de la France. L'influence anglaise, c'était déjà fini, les Anglais tenaient la police, parce que ce sont de bons policiers, mais c'est tout. Il y avait bien un collège anglais, ce n'était rien du tout. C'était absolument l'influence française, de nombreuses institutions, un hôpital français, des écoles...

Missions françaises d'Égypte

C'était la langue surtout, la langue française qui dominait. On parlait français partout où on allait, dans les magasins, même dans les petites boutiques comme les confiseries. Les familles qui avaient des gouvernantes, c'était des gouvernantes françaises. Parfois il y avait des Anglaises, mais alors ça c'était par snobisme. Beaucoup de troupes de théâtre françaises venaient, parce que c'était très rentable. J'ai vu toutes les actrices de ce temps, à Alexandrie. On allait aussi beaucoup au cinéma, et c'était des films français la plupart du temps.

31 Et il y avait une quantité d'écoles où l'enseignement était en français. Il y avait le lycée français, où on ne nous envoyait pas, nous les filles «bien », parce que c'était un petit peu libéral : pensez, il n'y avait pas de messe tous les jours. Et puis ma famille avait demandé une documentation, et ils avaient été très choqués: pour l'internat, on précisait: "chemises de nuit ou pyjamas ». Pyjamas ?! Le lycée français était une très bonne école, mais ils n'ont pas voulu, à cause des pyjamas... Les filles «bien » comme moi, nous devions aller chez les « Mères ». Les " Mères de Dieu » - où je suis allée - et les «Dames de Sion » ou le "Sacré Cœur ». Il y avait une succursale à Mansourah, une autre au Caire : ils ne lâchaient rien... Naturellement, c'était très cher. Les autres écoles, les «Sœurs de la Charité », les «Sœurs de Saint Joseph », c'était pour le "peuple ». C'était des « Sœurs », pas des « Mères ». Il y avait aussi des « Sœurs » chez nous, mais c'était des domestiques, la sœur cuisinière, la sœur lingère, la sœur tourière... Le matin, pour nous réveiller, une Mère passait avec un genre de castagnettes et criait : «Vive Jésus et Marie! " Nous devions répondre : «À jamais dans mon cœur ! Parfois nous ne répondions pas. Alors elle recommençait : « Vi-ve Jé-sus-zé-Marie !!! » « À jâmais-dans-mon-cœur !!! » Après ça on s'habillait, on mettait un voile blanc qui pendait jusqu'aux hanches, et on descendait à la messe. Pour se lever ou se mettre à genoux, tout était réglé avec une castagnette. Et le prêtre faisait les sermons. Tous les matins, et tous les après-midi au salut. Et puis le catéchisme... Et puis au début de l'année, la retraite... Alors ça, c'était... moyenâgeux, on lisait des choses saintes, trois fois par jour on allait à l'église et le prêtre nous parlait... La retraite ! C'était é-touf-fant !

33 Je rêvais d'aller étudier, de m'instruire. Je voulais être avocate. Ma mère m'a dit une chose merveilleuse : «Tu seras parfaite, tu insultes tellement bien les gens quand tu es fâchée !» À cette époque, c'était défendu, c'était impensable pour une femme d'exercer 
une profession. Même si elles avaient besoin de travailler, il ne fallait pas. Et puis d'ailleurs elles étaient illettrées pour la plupart. Quand elles avaient fait le lycée, elles savaient le français, elles savaient un peu pianoter... elles étaient bonnes à marier. Une fois mariées, elles faisaient une vie mondaine...

J'ai fait le baccalauréat là-bas, en Égypte, et puis j'ai enseigné à l'école. Mon frère était resté à Athènes pour faire ses études au lycée léonin, tenu par des religieux. Et lorsqu'il a fini le lycée, il a été à Grand, parce qu'il y avait une tradition dans la famille: les garçons allaient tous en Belgique - à Gand, à Liège, ou à Gembloux, pour ceux qui faisaient des études d'ingénieur agronome -, sauf ceux qui faisaient les Beaux Arts, qui allaient à Paris. Quelques-uns allaient aussi en Allemagne, des chimistes. Panagos aurait très bien pu faire ses études à Athènes, ç'aurait été deux fois moins cher. Non, il fallait aller à Gand, les garçons, chez nous, allaient à Gand...

e me suis donc mise à travailler - et j'ai toujours travaillé depuis, j'ai toujours été indépendante, depuis l'âge de seize ans. En ce temps-là, j'avais huit livres... et sept partaient pour Gand. Je gardais une livre pour prendre le tramway, pour acheter une paire de bas, des choses comme ça. Puis mon frère est revenu de Gand à Athènes et ma mère est allée le rejoindre. Il n'avait pas encore de travail, il venait de s'installer. Je suis donc restée encore deux ans en Égypte, chez ma tante, à travailler pour envoyer les sept livres. Et je suis revenue vers 1932/33.

Retour à Athènes

J'étais très contente de rentrer en Grèce. Parce que j'avais beaucoup souffert à l'école, je ne pouvais pas supporter leur mentalité. J'étais gosse, mais ça me mettait hors de moi. Et après, quand j'étais "jeune fille du monde », je ne pouvais pas supporter leur mentalité non plus, leur snobisme, leur distinction de classes. En Grèce, mon Dieu, on pouvait être ce qu'on était, ne pas feindre, ne pas tâcher d'être autre chose. Il n'y a jamais eu une telle différence de classes, un tel décalage. En Grèce, on formait son cercle comme on voulait, on n'était pas obligé d'être dans leur cercle. Les filles travaillaient - ce qui n'était pas admis en Égypte - dans les bureaux, dans les administrations, elles étudiaient, elles devenaient, je ne sais pas moi, avocates.

À Athènes, j'ai d'abord été dans un ministère, et puis j'ai travaillé pour un bureau spécialisé dans les traductions techniques. J'y allais l'après-midi, deux heures, et le reste du temps je traduisais à la maison. Quand j'avais passé le baccalauréat, je devais parler deux langues étrangères, et j'avais choisi le grec et l'italien. Mais c'était surtout les Français qui m'intéressaient. Ma culture était française, absolument. Je pensais en français. Le français, c'est ma langue.

À cette époque, tous les dimanches, nous nous réunissions chez Yannoulis Bonis ${ }^{7}$. Bonis était un homme extraordinaire à tous les points de vue, comme on n'en fait plus. Le vrai monsieur du siècle passé, tellement raffiné, très intellectuel, très musicien, s'occupant de littérature... Ce n'était pas une famille fortunée, il avait fait son droit, et toute sa vie il a travaillé dans une entreprise à Athènes, il venait à Mykonos en été. Il éditait alors les Mykoniatika Chronica : il y avait un petit groupe de gens qui écrivaient, toujours sur Mykonos - parce que c'étaient des chauvins terribles, les Mykoniates! Ils se réunissaient tous les soirs dans un café. Et le dimanche, chez lui, il y avait quelques jeunes femmes, et puis des jeunes gens, et tous ces gens étaient vraiment de qualité, il n'y avait aucune banalité... Et on parlait, et on parlait... C'était d'un charme... On parlait de tout, de Mykonos surtout, et de politique, beaucoup, parce que nous étions tous du même bord. De littérature aussi. 

puis ils étaient revenus en Grèce. C'était un type d'un humour fantastique. Ce qui était drôle, c'est qu'il pouvait écrire dans deux langues : le grec savant - la katharévoussa qu'on enseignait alors dans les écoles, ou le grec de Mykonos. Mais la langue que nous parlons, ce que nous appelons aujourd'hui dimotiki glossa, il n'était pas capable de l'écrire.

D'Eupatoria à l'atelier de Bourdelle...

\section{plus tard.}

41 des ports très facilement accessibles, il y avait tout un commerce. Sa famille était originaire d'Épire, d'un village qui s'appelle Vassiliko. Ils habitaient Eupatoria, mon beau-père avait une flotte et une industrie de boutargue, de produits de pêche. Et il avait aussi de petits immeubles - ce qui faisait beaucoup, paraît-il, pour les touristes qui venaient l'été pour les bains : Eupatoria, c'était un lieu de villégiature. Il y avait deux chambres, un samovar, deux chambres, un samovar, et ainsi de suite. Dans sa famille, ils ne savaient pas du tout le grec, à l'exception de ma belle-mère, parce que son père était pope. Elle était la sœur de Cheimonas, le fameux peintre. Il était très apprécié, et il parait qu'à l'Ermitage il y a des tableaux de lui. Et mon mari me racontait que lorsqu'ils étaient gosses, ses deux frères et lui, on leur demandait: "Qu'est-ce que tu veux faire ? » Alors l'aîné disait : «Je veux être médecin ». Le troisième disait : « Moi je veux être ingénieur ». Et Télis ne répondait pas. Ils en avaient conclu qu'il voulait être commerçant! Entre temps, chaque fois que Cheimonas sortait pour peindre, il s'asseyait près de lui, et il regardait, il regardait... Mais il ne disait rien. Alors ils en avaient conclu que ce serait un grand commerçant ! Et l'aîné est devenu chirurgien, le dernier est devenu ingénieur, Télis n'est pas devenu commerçant...

Il est venu en Grèce en 17, il avait quinze ans. Et ils sont allés à Égine, parce que c'était le littoral, la mer. Ils n'avaient pas pu aller dans une ville. Et puis quand ça s'est un peu calmé en Russie, ils sont retournés à Eupatoria. Mais peu de temps après, ils ont dû partir définitivement. Pourtant toute sa vie, le père de Télis a été fanatique de la Russie, bien qu'il en ait été chassé. Et Télis aussi avait été très marqué par la Russie. Il est parti quelques années plus tard à paris, avec cent francs en poche. Il voulait allait étudier aux Beaux-arts, il n'avait pas d'argent pour payer son passage. Alors un agent de voyages qu'il connaissait lui a donné un billet... pour enfant! À Paris, il y avait des Grecs de partout. Le peintre Papageorge, par exemple, était d'Égypte, il parlait parfaitement le français. Mais ceux qui ne connaissaient pas le français étaient un petit peu perdus. Ils étaient très jeunes, ils formaient un noyau. Il y avait le sculpteur Dimitriadis, qui regroupait autour de lui les jeunes Grecs qui étudiaient les arts, qui les protégeait. Et puis des mécènes, des gens comme Zervos, Tériade ${ }^{8}$. Télis a travaillé, il a d'abord commencé par poser comme modèle pour Bourdelle.

Je l'ai connu à Mykonos, en 1946, l'été... Quelques jours après j'ai dit à mon frère : « Tu sais, ce type-là, je vais l'épouser. » Il m'a répondu : « Tu es folle ! ? Mais tu ne le connais pas! Et s'il te coupe en morceaux ?! » Je lui ai dit : «S'il me coupe en morceaux, c'est moi qu'il coupe, ce n'est pas toi ! » Nous nous sommes mariés à Mykonos. Et j'avais une bonne, Irini, depuis très longtemps. Lorsque nous sommes rentrés, Irini me dit: «Montez là-haut! Montez là-haut!!!» «Mais fiche-nous la paix, pour l'amour du 
Ciel !» Elle jugeait qu'il fallait aller consommer le mariage ! Et elle était choquée parce que le soir je suis allée à la taverne pour dîner...

44 a commencé à fleurir, je me suis mise à tricoter pour un magasin. De huit heures du matin, jusqu'à quatre ou cinq heures, je traduisais vingt pages, et je tapais à la machine. Et de cinq heures à minuit, je tricotais. Comme cela, ma fille a pu faire ses études à Paris, j'avais juste la somme qu'il fallait lui envoyer chaque mois. Elle avait une facilité extraordinaire pour les langues, et j'avais dit à Télis : « Il faut l'envoyer à Paris, à l'École des Traducteurs ». Et lui : "Mais tu es folle, comment est-ce qu'on va l'envoyer à Paris? » J'ai répondu: «Je m'en charge.» Et, antre autres arguments, mon mari a ajouté : «Mais tu sais que si elle va à Paris, elle ne va plus revenir?" «Je le sais très bien, elle ne va plus revenir. Et alors? Je l'espère pour elle !»

La pluie d'or du tourisme

Tous les étés, nous retournions dans la maison d'Ano Mera. À Mykonos, après la Première Guerre mondiale, il y avait eu un changement de mentalité, il n'y avait plus ce paternalisme, les gens commençaient à devenir indépendants, les carrières étaient plus ouvertes, les garçons allaient à l'école. Ils n'étaient plus absolument dépendants de ces grosses fortunes comme avant. Ils avaient commencé à s'expatrier, à aller en Amérique pour travailler, et puis les cousins suivaient. Leur profession initiale, c'était toujours laveur de vaisselle. Dès qu'ils avaient un peu d'argent, ils revenaient, ils avaient une nostalgie spéciale de Mykonos. Ils rafistolaient leurs maisons, ils se mariaient.

Je me rappelle... il y avait une taverne, cinq ou six tables, le patron était tellement amusant que nous y allions tous les soirs. Et nous le taquinions. Il était resté vingt ans en Amérique, et il était revenu avec... vingt dollars en poche, vingt dollars! C'était amusant, parce qu'il se chamaillait tout le temps avec sa femme, et puis il avait un rire très particulier. Alors sa femme nous a raconté un jour très sérieusement : « Moi je l'ai épousé, espérant qu'après vingt ans d'Amérique, il aurait de l'argent. Et après vingt ans, il a ramené vingt dollars, et qu'est-ce qu'il me restait à moi ?... Son rire, rien d'autre... »

Les Mykoniates ont toujours été très particuliers. Les femmes, par exemple, elles sont... terribles! Je ne sais pas à quoi ça tient, cette liberté des femmes. Parce qu'après tout, c'est une île parmi des centaines d'autres. Pour les femmes de marins, c'était un petit peu normal, parce que leurs maris n'étaient pas là. Laissons les marins, moi je me souviens très bien des paysans : tous les dimanches, ils descendaient en ville, et puis ils se rencontraient au café, à la taverne, pour discuter, on va louer tel champ, à tel prix... Eh bien les femmes y allaient aussi. Chose extraordinaire pour n'importe quel petit pays en Grèce, elles s'asseyaient à la taverne avec les hommes ! Et si la femme n'était pas là, le type disait toujours: « $\theta \alpha \rho \omega \tau \eta \sigma \omega \tau \eta \nu \kappa U \rho \alpha \gg{ }_{\text {(je }}$ demanderai à la patronne). Les autres îles, disons Tinos, Naxos, sont beaucoup plus grandes. Nous, c'est un tout petit noyau, alors... le père parti, la femme avait la responsabilité de tout. C'était absolument un matriarcat. Et qui continue, dans un certain sens. Elles sont plus combatives, elles prennent les choses en mains. Et puis au fond, ça arrange les hommes. Ils laissent faire : elles font bien !

Et Mykonos a toujours été une île très libre, à un point inimaginable. L'union libre, par exemple, était tout à fait admise. Des bâtards, on pouvait en avoir, ce n'était pas une

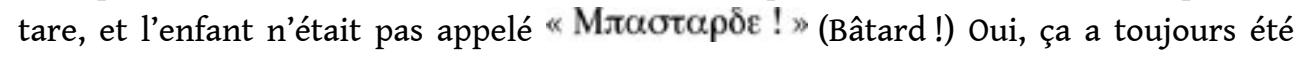
comme ça, je me souviens, étant gosse, d'unions libres. Pourquoi ? Parce que le divorce 
coûtait cher. Dans une autre Cyclade, on aurait égorgé une femme qui vivait avec un homme sans être mariée, on l'aurait mise tout à fait en marge. Ici, c'était très admis.

Ou bien par exemple une putain... Ça n'a jamais été une tare. La fameuse Floureza qui, très vieille, était assise sur le port et cirait les souliers, c'était une putain officielle. Lorsqu'elle était très jeune, elle était domestique chez ma grand-mère. Elle s'est mariée, et elle s'est mise à faire ce métier. Elle a fait sept ou huit enfants. Et elle disait parfois : «Celui-là, le Yannos, mon préféré, j'ai dû le faire avec quelqu'un de très bien. » Et ils vivaient tous ensemble, avec son mari.

Le tourisme a commencé vers 1928/30. Certaines familles - grecques - amies de familles de Mykonos sont venues ici, elles ont loué des maisons, elles sont revenues. Nous avions des amis d'Athènes qui ont acheté des terrains, par exemple Éléni Vlachos, qui avait le journal Kathimérini. Elle a fait venir beaucoup de gens du journal, et de son milieu. Alors avec eux, d'autres venaient, quelques-uns d'Alexandrie. Quelques peintres aussi, Athineous, Ianoukakis. Il y a eu le Délos, le premier hôtel. Et quelques étrangers ont commencé à venir, avec des amis grecs. Vers 1935, le vieux Cambanis, qui était député, a fait construire l'École des beaux arts, destinée à héberger des peintres. De temps à autre, un bateau de croisière s'arrêtait. Alors les villageois dressaient des tables, près du port, les femmes étalaient leurs tissages, elles avaient confectionné des sortes de grands draps de bain pour s'étendre, qui déteignaient un peu... Les touristes passaient, ils achetaient.

À Délos', on a commencé à mettre de l'ordre, parce qu'auparavant on faisait ce qu'on voulait. Quand le musée n'était pas encore bâti, chacun pouvait emporter ce qu'il souhaitait. C'est pour ça que Mykonos est tellement blanche, parce qu'ils avaient le marbre à profusion, ils en remplissaient des caïques, ils le transformaient en chaux. Et quantité de maisons mykoniates ont été faites avec des marbres de Délos. Ils faisaient venir des colonnes... Regardez les marches des vieilles maisons : quelques-unes ont une marque en plomb, au milieu : c'était là où il y avait les statues ${ }^{10}$. Et au début du siècle, il y a eu à Mykonos certaines fortunes qui se sont faites sur le trafic d'œuvres de Délos... Tel sous-sol était plein de statues et de marbres rapportés la nuit, et tout cela repartait vers les musées d'Europe...

Mais le tourisme, avant-guerre, n'avait encore aucune influence sur l'économie de l'île. Les maisons étaient louées pour rien. Après la guerre, la folie du tourisme est venue tout à coup, comme ça. Je pense que les gens en avaient par-dessus la tête de cette guerre et de cette occupation qui avaient été terribles, et ils voulaient quitter un peu Athènes. Alors très vite tout a renchéri, il y a eu de nouveaux commerces, de nouvelles industries. Ce sont les gens du cru qui ont saisi la balle au bond. Les Athéniens sont venus bien après. Tout de suite, les Mykoniates ont compris. Ils ont commencé à louer des chambres, ils ont apporté de nouvelles choses, ils ont agrandi les magasins, construit de nouvelles boutiques, des restaurants, des tavernes...Et il n'y a pas eu d'ascensions spectaculaires, mais petit à petit... Les villageois étaient heureux comme tout. Ils avaient toujours été hospitaliers, mais alors là !

51 Les Mykoniates avaient ça dans le sang: ils sont très "réceptifs ", au sens profond du terme, ils s'adaptent facilement. Et ils ont toujours tout admis. En riant. Quant à leurs défauts actuels, ils n'avaient pas eu l'occasion de sa manifester: ils sont rapaces, ils aiment beaucoup l'argent. Pour leurs enfants, aujourd'hui, c'est uniquement une éducation utilitaire. Autrefois, il fallait l'instruire, le garçon, pour qu'il soit mieux que soi. Maintenant... pourquoi faire? Dans toute la Grèce, les valeurs matérielles ont pris 
le pas sur les valeurs plus « spirituelles ». Mais c'est plus fort à Mykonos, parce qu'il y a eu un changement si rapide... Il y a des magasins qu'on loue 300000 drachmes ${ }^{11}$ par mois, pour toute l'année. Mon pharmacien me racontait... il a une maison, et en bas, sous l'escalier, il y a une petite porte qui donne sur la rue - c'était pour mettre les pots de chambre. Quelqu'un est venu pour lui louer ça, pour vendre des colifichets. Il lui a demandé : « Mais comment vas-tu faire ? On ne peut pas entrer là-dedans, même sur le ventre ! » « Je n'ai pas besoin d'entrer. J'exposerai dehors, et le soir je remiserai là. »

Et ils sont snobs, tous; Mégalomanes : «Tu sais qui je suis, moi ? Ça, c'est terrible... Leurs maisons, leurs voyages... en Russie, partout. Tous les hivers, ils voyagent, tous, une vraie compétition. Et ils vont très souvent en Amérique, parce qu'ils ont des parents là-bas. Et ils se vantent... Enfin, comme on dit à Mykonos :

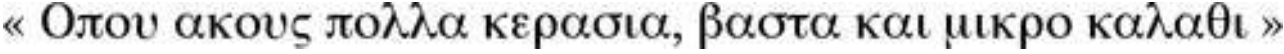

(Si on te raconte qu'il y a beaucoup de cerises quelque part, tu ne prends qu'un petit panier...)

(Récit recueilli par Michèle Petit)

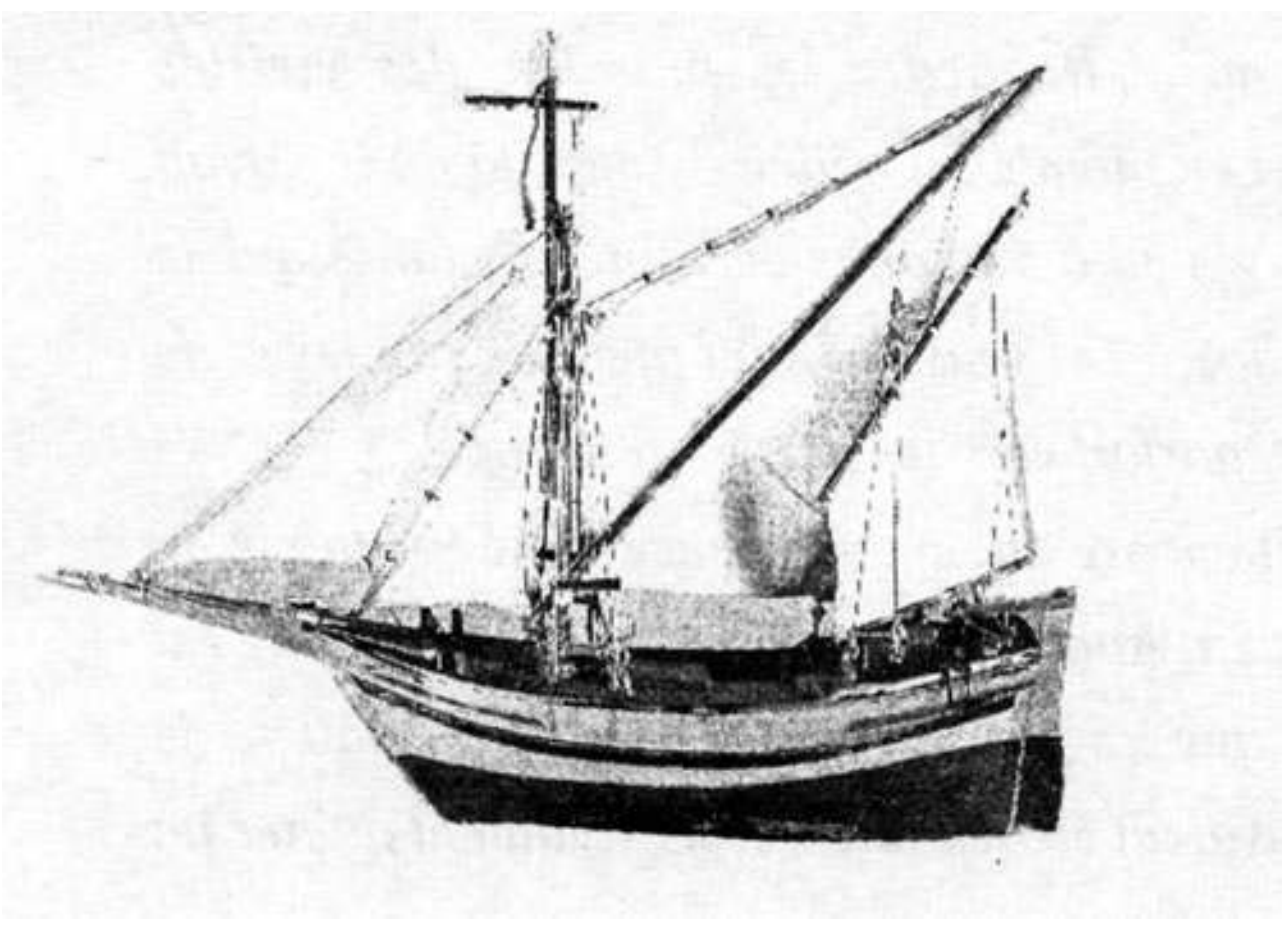

\section{NOTES}

1. Hameau situé au centre de l'île de Mykonos.

2. Dès le XIII ${ }^{\mathrm{e}}$ siècle, des praticiens vénitiens s'étaient taillé des domaines dans les Cyclades, et Mykonos fut sous administration directe de la République de 1390 à 1540 (Cf. Jean Baelen, Mykonos, chronique d'une île de l'Égée, Les Belles Lettres, Paris, 1964.) 
3. La famille Gripari s'était initialement établie à Sébastopol, où elle s'adonnait, sur une vaste échelle, à l'exportation de céréales et à l'importation de charbon. Quand Sébastopol fut transformé en arsenal, la fortune familiale fut convertie en avoirs fonciers et industriels. La propriété de Baranovka aurait été rachetée aux deux dernières descendantes de la famille princière Gagarine, établies sur la Côte d'Azur ; Située en Ukraine, près de Jitomir, cette propriété couvrait 35000 hectares, et comprenait des villages et un palais princier, l'une des plus importantes usines de porcelaine de Russie, une verrerie et une minoterie. Consul Général de Grèce et de plusieurs autres pays pour la Russie du Sud, Nicolas Gripari aurait pris la nationalité russe pour pouvoir l'acquérir (Cf.

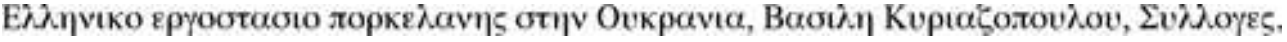
Athènes, 2/11/1983.) Selon Frosso Vassilikioti, les sœurs Gagarine auraient cédé d'autant plus volontiers leur propriété que l'on venait d'y procéder à la coupe du bois, et qu'il fallait, selon la réglementation en vigueur, attendre dix années avant de procéder à une nouvelle coupe. De par ses entrées à la cour, Nicolas Gripari eut vite fait d'obtenir une dérogation lui permettant de n'attendre qu'une année...

4. Les Ralli, originaires de l'île de Chio, sont parmi les premiers négociants grecs à s'installer en Angleterre au début du XIX ${ }^{e}$ siècle. Ils fondent alors une entreprise commerciale véritablement «multinationale », gérée familialement, qui comprend des branches en Angleterre, à Marseille, Odessa, Smyrne, Constantinople, Tabriz. En 1851, la société de Londres prend le nom de «Ralli brothers » et ouvre dans les années suivantes de nouvelles succursales aux Indes, où elle occupe une des premières places dans le commerce du coton, sur la mer Caspienne, à New York et en Égypte. Ils établissent une compagnie de navigation à Trieste, qui compte pour la première compagnie de navigation de l'Autriche-Hongrie, et relie bientôt les États-Unis à l'Angleterre, Marseille, Odessa, à l'Empire ottoman et aux Indes. En 1863, avec deux autres familles originaires de Chio, ils créent l'Imperial Bank à Alexandrie. Dans l'entredeux-guerres, «Ralli brothers » poursuit ses activités dans le commerce et la finance puis, avec la fin de l'Empire britannique, ses activités déclinent et le groupe perd son nom lors d'une fusion financière ( $C f$. Sturdza, Grandes familles de Grèce, d'Albanie et de Constantinople, Paris, 1983, p. 386 à 394 et Christos Hadziiossif, « Banques grecques et banques européennes au XIX ${ }^{e}$ siècle : le point de vue d'Alexandrie ", in Georges Dertilis et al., Banquiers, usuriers et paysans. Réseaux de crédit et stratégies du capital en Grèce (1780-1930), Fondation des Treilles/La Découverte, Paris, 1988. Cf. aussi « Alexandrie entre deux mondes ", Revue de l'occident musulman et de la Méditerranée, 46, 1987, Edisud, et les travaux de Robert Ilbert.)

5. Famille originaire de l'île de Syra. Emmanuel Bénakis (1843-1929), après avoir fait fortune dans le grand commerce à Alexandrie, s'établit à Athènes dont il devint maire en 1914. Il y fonda des hôpitaux et un musée qui porte son nom.

6. Famille patricienne de l'île de Chio, dont les membres se dispersèrent après 1821 à Trieste, Marseille et Alexandrie, où ils s'adonnèrent au commerce et à la finance fondant en 1870 la « National Bank of Egypt ». Miké Salvagos fut président de la communauté grecque d'Alexandrie de 1919 à 1948. Son conseiller était un ancien ministre de Vénizelos, Georges Roussos.

7. Yannoulis Bonis (1878-1960) a édité de décembre 1933 à août 1935 les Chroniques

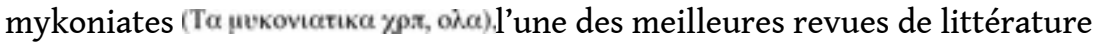
« provinciale » d'avant-guerre. Des nouvelles publiées dans les Chroniques mykoniates ont été rééditées récemment par Panagiotis Kousathanas 


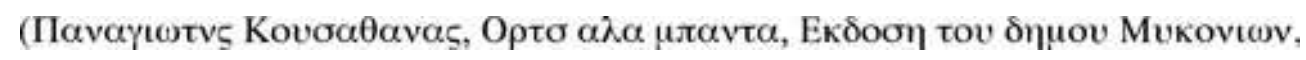
Mikonos, 1986.)

8. Deux éditeurs d'art qui ont contribué de façon décisive à imposer l'art contemporain. Christian Zervos (1889-1970), après avoir passé le début de sa vie à Alexandrie et à Marseille, vint à Paris où il fonda en 1926 la revue Cahiers d'art, qui paraîtra jusqu'en 1960. Avec sa femme Yvonne Zervos, il a joué un rôle déterminant dans la formation du goût actuel, tant par des publications que par des manifestations artistiques. Né dans l'île de Mytilène, Tériade (1897-1983) arriva à Paris en 1915. Il créa avec Albert Skira la célèbre revue Minotaure et en assura la direction artistique jusqu'en 1936 ( $C$ ). Arturo Schwartz, Les onze Minotaures, FMR, 13, mars/avril 1988 et Regards sur Minotaure, la revue à tête de bête, catalogue de l'exposition réalisée au Musée d'Art Moderne de la Ville de Paris au printemps 1988, Skira, Genève). Les Minotaure ont été réédités récemment par Skira, en trois volumes. Tériade publia également, de 1937 à 1960, la belle revue Verve, ainsi que vingt-six grands « livres de peintres ».

9. «L'île sacrée »... qui se couvrit de fleurs et d'or quand la déesse Léto y accoucha d'apollon, est distante de quelques kilomètres de Mykonos. Pratiquement abandonnée au début de notre ère, elle ne retrouva jamais l'activité commerciale ou politique qu'elle avait connu dans l'Antiquité. Elle reçut la visite des Chevaliers de Saint Jean, des Vénitiens, de nombreux voyageurs dont André Thevet, moine géographe de retour de Terre sainte, des Turcs, de pirates qui s'y réfugiaient, de pillards de toute provenance... et des paysans de Mykonos qui y paissaient leurs troupeaux. L'île appartient administrativement à Mykonos depuis 1841. Le dégagement du sanctuaire d'Apollon par les archéologues français commença en 1873, et les travaux s'intensifièrent entre 1904 et 1914

10. En 1700 , lors de son voyage, Tournefort notait déjà : « On monte jusqu'au sommet de la colline par un escalier de marbre, dont la plupart des marches ont été emportées à Mycone pour faire des appuis de fenêtres (...) On rencontre quelques restes de lions de marbres tous en pièces. » Quant à la statue d'Apollon, auquel Délos était vouée... elle aurait été vue entière en 1418 , au milieu du XIv ${ }^{e}$ siècle ne subsistait que le tronc, et en 1630, ce tronc était coupé en deux... (Cf. Joseph Piton de Tournefort, Voyage d'un botaniste, T. 1, La Découverte, Paris, 1982, pp. 254 à 258.) Délos est aujourd'hui l'île des dieux sans têtes, puisque les pillards de tous lieux les décapitaient pour séduire musées et collectionneurs.

11. Environ 12000 F (été 1990).

\section{RÉSUMÉS}

Ce « carnet de terrain» reprend des passages d'un récit de vie inédit, fruit de longs entretiens avec Frosso Vassilikioti, dont la famille, originaire de l'île de Mykonos dans les Cyclades, s'illustra dans la circulation des biens comme dans celle des idées, des rivages sud de l'Europe à la Russie et au Proche-Orient. Ces passages sont précédés d'extraits des notes de Michèle Petit qui a recueilli ce récit, privilégiant les méthodes qui donnent leur juste place aux acteurs singuliers composant le social. 
The Aegean diaspora : a woman's life story

This «fieldwork notebook» reports some passages of an hitherto unpublished life story, resulting from extensive interviews with Frosso Vassilikioti, whose family, originating from the Mykonos island in the Cyclades, wan fame though goods as well as ideas circulation from the southern Europe shores to Russia and the Middle East. These passages aree preceded by extracts of notes written by Michèle Petit who has collected this life story, emphasizing the methods which allow not to dissociate the social context from the individuals who are part of it.

\section{INDEX}

Keywords : Diaspora, élites, life story, fieldwork notebboks, Medeterranean shores and islands, Greece, Russia, Egypt

Mots-clés : Carnets de terrain, récit de vie, Méditerranée et îles, Grèce, Russie, Égypte

\section{AUTEUR}

\section{FROSSO VASSILIKIOTI}

D'une famille d'intellectuels et d'hommes d'affaires de la diaspora grecque originaire de l'île de Mykonos dans les Cyclades, elle a été traductrice. Elle est aussi l'auteur d'une « littérature orale » qui donne voix à la mémoire des lieux où elle a habité. 